\title{
Carateres de seleção do toiro de lide para a tourada à corda dos Açores
}

\author{
Correia, P. ${ }^{\circledR}$; Baron, E. ${ }^{1}$ e Moreira da Silva, F.
}

Reprodução Animal. Departamento de Ciências Agrárias. Universidade dos Açores. Angra do Heroísmo. Portugal.

\section{PALAVRAS CHAVE ADICIONAIS}

Bravura.

Carateres comportamentais.

\section{ADDITIONAL KEYWORDS \\ Aggressiveness. \\ Behavioral traits.}

\section{INFORMACIÓN}

\section{Cronología del artículo:}

Recibido/Received: 3.3.2014

Aceptado/Accepted: 27.11.2014

On-line: 16.3 .2015

Correspondencia a los autores/Contact e-mail:

correia@uac.pt

\section{RESUMO}

Com o objetivo de identificar caraterísticas com interesse na seleção do toiro de lide para as touradas à corda, foi elaborado um inquérito, incluindo 38 questões, tendo sido inquiridos, no período de janeiro a março de 2013 , todos os ganadeiros $(n=18)$ inscritos na Associação Regional de Criadores de Toiros da Tourada à Corda. Os resultados foram submetidos a análise estatística com um teste não paramétrico de qui-quadrado por simulação Monte Carlo (MMC-10 000 bootstraps) ( $\leq \leq 0,05)$. Os resultados obtidos evidenciaram que todos os ganadeiros definiram elevada mobilidade para os toiros das touradas à corda (pontuações $\geq 3)$, e $88,89 \%(p \leq 0,05)$ destacaram esta caraterística como uma das mais importantes para a seleção. Para os ganadeiros os toiros têm de responder rapidamente aos estímulos de investida e com temperamento altamente agressivo e combativo. Esta caraterística foi apontada como uma das mais importantes na seleção do toiro de lide para a tourada à corda $(94,44 \%-p \leq 0,05)$. Os toiros devem estar atentos ao que ocorre à sua volta, $(94,44 \% ; p \leq 0,05)$. Os resultados ainda aferiram que todos os ganadeiros destacaram a resistência como um caracter a selecionar nos animais. Ficou demonstrado que os ganadeiros valorizam a melhoria do desempenho dos toiros ao longo de uma tourada à corda. Através da avaliação dos itens REG34, REG36, REG35 e REG37, ficou evidenciado que a regularidade dos desempenhos dos toiros nas várias touradas é um caráter muito levado em consideração pelos ganadeiros. No final foram identificados e definidos sete caracteres: mobilidade; capacidade de arremeter; capacidade de fixar; codícia; resistência; crescer e regularidade. Os caracteres foram organizados numa escala de avaliação para o seu uso na seleção do toiro de lide para a as touradas à corda.

\section{Selection traits of lidia cattle for Azorean street bullfighting}

\section{SUMMARY}

In order to identify and define selection traits for the breeding of bulls destined to the typical Azorean street bullfighting (tourada à corda), a 36-item questionnaire was submitted to all members of the Associação Regional de Criadores de Toiros da Tourada à Corda $(n=18)$ between January and March, 2013. Results were analyzed with SPSS, using chisquare nonparametric testing with Monte Carlo simulation (MMC-10000 bootstraps), with a significance value of $p \leq 0.05$. The obtained results showed that every breeders define high mobility to the bulls of Azorean street bullfighting (scores $\geq 3)$, and $88.89 \%(p \leq 0.05)$ highlighted this as one of the most important characters for selection. For the breeders, the bulls need to respond quickly to the stimulus with aggressiveness and high capacity to fight $(100 \%)$. This character was indicated as one of the most important to the bulls selection for the Azorean street bullfighting $(94.44 \% ; p \leq 0.05)$. The bulls should be alert to the surrounding area $(94.44 \% ; p \leq 0.05)$. The results also showed that all breeders highlighted the resistance as a character to select in these type of animals. The bullfighting breeders prefer animals who improve the performances in the bullfight. In the evaluation of the questionnaire items REG34, REG36, REG35 and REG37, the breeders showed it is very important to the bull show similar performances in all the bullfights. In the end, seven major traits were identified and defined: mobility, charging aptitude, focus, aggressiveness, endurance, rise and consistency. A scoring table is proposed as an animal evaluation tool for use in Lidia cattle selection for Azorean street bullfighting.

\section{INTRODUÇÃO}

A seleção direcionada essencialmente para aspetos comportamentais, nomeadamente a bravura, diferencia a raça Brava de Lide das demais raças de bovinos, onde os carateres de natureza física (produção de carne ou de leite) são o objetivo da seleção. O desenvolvimento da raça Brava de Lide inicia-se, exatamente, pela procura de animais para a realização de diferentes manifestações culturais taurinas e, hoje em dia, carateriza-se por um padrão etológico bem definido (Gaudioso e Sánchez, 1987). Apesar de, na génese da raça, ter sido tida em conta, essencialmente, a obtenção de animais agressivos para as corridas de toiros em praças, outros objetivos de seleção surgiram, nomeadamente aplicados a festejos de natureza popular e.g. largadas, entradas de toiros, recortadores, encierros, touradas à corda, etc. Os carateres comportamentais do toiro de lide, assim como as formas de os medir, têm vindo a ser estudados por vários autores (Sánchez 
et al., 1990; Gaudioso et al., 1993; Garcia e Almenara, 2004; Garcia-Schneider, 2008; Calvo, 2010; AlmenaraBarrios e García, 2011). Vários resultados desses estudos têm sido aplicados no melhoramento genético de ganadarias bravas, nomeadamente os relativos à heritabilidade das componentes da bravura (González et al., 1994 e Silva et al., 2006), ou à fisiologia do toiro de lide (Garcia-Schneider, 2008; Gil-Cabrera, 2013).

Na ilha Terceira (Açores), o toiro e as touradas ganham um especial impacto sociocultural e económico, com a tourada à corda, manifestação tauromáquica única no mundo. Este festejo carateriza-se por uma forte regulamentação, revista pelo Decreto Legislativo Regional n. . 37/2008/A, com uma última alteração imposta pelo Decreto Legislativo Regional n. ${ }^{\circ}$ 13/2012/A. Os toiros são soltos nas ruas, embolados e com uma corda atada ao pescoço. Um grupo de dez homens, chamados pastores, controla o toiro, segurando na corda. A rua transforma-se em arraial de intenso convívio social, onde homens conhecidos por capinhas enfrentam o toiro, a corpo limpo ou com trastes de capear, com um guarda-sol ou uma simples manta. Nestes eventos, os toiros não são feridos, sendo corridos várias vezes ao longo da vida e, por vezes, ganhando fama na sociedade civil local. Atualmente, as touradas à corda foram expandidas para outras ilhas açorianas como Graciosa, São Jorge, S. Miguel e Pico, que passaram a ter ganadarias locais.

As ganadarias da ilha Terceira executam tipos de seleção distintos, que variam consoante o tipo de manifestação a que os animais se destinam (lide em praça, ou tourada à corda). São inexistentes os documentos que abordam a temática da seleção do toiro de lide para a tourada à corda de forma organizada. No entanto, segundo Merelim (1986), Bruges (1997) e Costa (1999) a seleção do toiro de lide para a tourada à corda sempre esteve associada em certo grau à seleção tradicional destes animais para a lide em praça. Manuscritos datados de meados do século passado relatam a existência de uma seleção empírica específica para a tourada à corda, tendo como objetivo a obtenção de animais altamente agressivos, temperamentais e imprevisíveis (Silva, 2011).

Segundo Ferreira (2007), essa seleção empírica do toiro de lide para a tourada à corda, que ainda hoje se pratica com maior intensidade, tem importância na

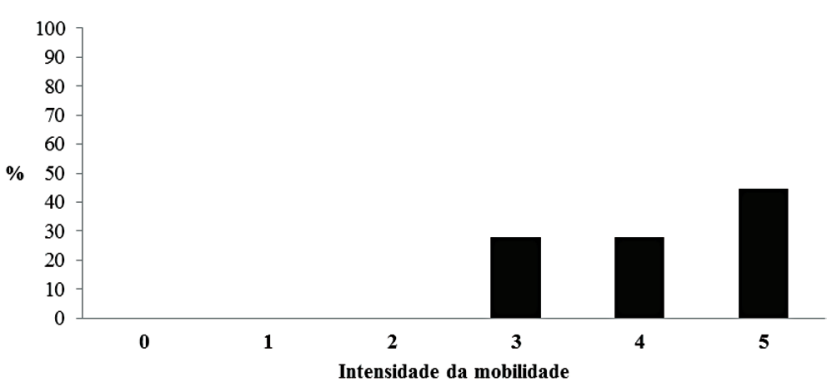

Figura 1. Intensidade da mobilidade do toiro na tourada à corda $(\mathrm{MOB} 1)$, sendo 0 intensidade nula e 5 intensidade extrema (Mobility intensity of bull in the Azorean street bullfighting (MOB1) 0 being null and 5 extreme).

conservação de recursos genéticos açorianos, podendo ter sido a responsável pela preservação da linha designada, pela Secretaria Regional da Agricultura e Florestas (2010), como Brava dos Açores.

É nesse sentido que, esta investigação teve como objetivos, documentar de forma organizada caraterísticas que os ganadeiros utilizam para a seleção do toiro de lide para a tourada à corda, bem como, iniciar o desenvolvimento de uma escala de avaliação, que no futuro poderá apoiar o melhoramento genético para a tourada à corda.

\section{MATERIAL E MÉTODOS}

Atendendo à relação existente entre a seleção do toiro de lide para corrida de toiros em praça, e a praticada para a tourada à corda baseada na informação de Merelim (1986), Bruges (1997) e Costa (1999), foi desenvolvido um inquérito composto por 6 ítens de caracterização demográfica (sexo, localização, idade, profissão, escolaridade, anos na atividade de ganadeiro) e 38 itens sobre o comportamento do toiro de lide. Nesse sentido, as questões foram delineadas mediante comportamentos positivos e negativos evidenciados pelo toiro de lide quando lidado em praça apresentados por García e Almenara (2004). Ainda foram levados em consideração aspetos específicos das touradas à corda (e.g. desempenho do toiro entre touradas à corda, busca pelo local das gaiolas, tentativa de fuga do local da tourada, intensidade da investida a estímulos fixos e móveis). Todas as perguntas relacionadas com o

Tabela I. Grupos que constituíram os inquéritos, com o número de questões por grupo (N) e os aspetos avaliados em cada grupo (Groups issues that constituted the questionnaire, with the number of questions per group $(\mathrm{N})$ and the assessment objectives in each group).

\begin{tabular}{|c|c|c|}
\hline Grupos & $\mathrm{N}$ & Aspetos abordados nas questões dos distintos grupos \\
\hline 1 - Mobilidade (MOB) & 5 & $\begin{array}{l}\text { Locomoção do toiro, nomeadamente, tipos de andamentos (passo, trote ou galope), velocidade } \\
\text { e coordenação de movimentos). }\end{array}$ \\
\hline 2 - Capacidade de arremeter (ARR) & 5 & Aspetos da arrancada para a investida (García et al., 2003) \\
\hline 3 - Capacidade de fixar (CFX) & 6 & Capacidade auditiva e visual do toiro. \\
\hline 4 - Investida (INV) & 10 & $\begin{array}{l}\text { Ação da colhida do toiro tanto a estímulos móveis (capinhas) ou fixos (paredes, tábuas, etc.) } \\
\text { (García et al., 2003), e }\end{array}$ \\
\hline 5 - Resistência (RES) & 4 & Vigor e resistência física demonstrada ao longo da tourada à corda. \\
\hline 6 - Desempenho (DES) & 3 & Tendências da evolução do desempenho do toiro ao longo da tourada à corda. \\
\hline 7 - Regularidade (REG) & 5 & $\begin{array}{l}\text { Desempenho dos toiros nas diversas touradas à corda onde participam, assim como, perguntas } \\
\text { direcionadas à importância da regularidade do comportamento. }\end{array}$ \\
\hline
\end{tabular}


Tabela II. Avaliação de sinais positivos e negativos demonstrados pelos toiros nas touradas à corda em função das respostas dos ganadeiros (Evaluation of positive and negative behavioral traits in bulls to the Azorean street bullfights, made by the breeder's opinion).

\begin{tabular}{|c|c|c|c|c|c|}
\hline Acrónimo & & Descrição de itens avaliados no inquérito & $P$ & $\mathrm{~N}$ & I \\
\hline \multirow{3}{*}{ MOB } & 2 & Agilidade e velocidade como bons indicadores de mobilidade. & $100,0^{* *}$ & 0,0 & 0,0 \\
\hline & 3 & Deve revolver-se rapidamente à sucessão de passes dos capinhas. & $100,0^{* *}$ & 0,0 & 0,0 \\
\hline & 4 & Apresentar movimentos coordenados. & $100,0^{* *}$ & 0,0 & 0,0 \\
\hline \multirow{4}{*}{ ARR } & 6 & Arrancar sem hesitar aos estímulos de investida que recebe. & $100,0^{* *}$ & 0,0 & 0,0 \\
\hline & 7 & Tentativas de fuga do recinto da tourada à corda (arraial). & 0,0 & $100,0^{* *}$ & 0,0 \\
\hline & 8 & Busca incansável pelos locais das gaiolas no arraial. & 0,0 & $100,0^{* *}$ & 0,0 \\
\hline & 9 & Presença de sinais de temperamento agressivo e combativo. & $100,0^{* *}$ & 0,0 & 0,0 \\
\hline \multirow{5}{*}{ CFX } & 11 & A visão e audição devem persistir em tudo o que ocorre no arraial. & $94,4^{*}$ & 5,6 & 0,0 \\
\hline & 12 & Atenção única e exclusiva aos utensílios dos capinhas. & 61,1 & 38,9 & 0,0 \\
\hline & 13 & Passagens despercebidas pelos utensílios com a cara alta e sem ter intenção de os voltar a procurar. & 0,0 & $100,0^{* *}$ & 0,0 \\
\hline & 14 & Manter atenção aos capinhas enquanto investe aos utensílios que eles utilizam. & 72,2 & 27,8 & 0,0 \\
\hline & 15 & Caminhar sem prestar atenção e olhar em todas as direções. & 0,0 & $94,4^{*}$ & 5,6 \\
\hline \multirow{5}{*}{ INV } & 19 & Repetir as investidas sobre os capinhas. & $100,0^{* *}$ & 0,0 & 0,0 \\
\hline & 20 & Manter temperamento agressivo e combativo durante a investida. & $100,0^{* *}$ & 0,0 & 0,0 \\
\hline & 21 & Derrotar com a cabeça enquanto investe. & 50,0 & 50,0 & 0,0 \\
\hline & 22 & Curvar a cabeça durante a investida. & 0,0 & $94,4^{*}$ & 5,6 \\
\hline & 25 & Apresentar qualidades de animal toureável sobre as normas do toureio a pé (parar, templar e mandar). & 27,78 & $66,7^{*}$ & 5,6 \\
\hline \multirow{2}{*}{ RES } & 27 & Ter vigor e resistência de modo a transmitir emoção ao longo da tourada à corda. & $100,0^{* *}$ & 0,0 & 0,0 \\
\hline & 28 & Não apresentar esgotamento físico. & $100,0^{* *}$ & 0,0 & 0,0 \\
\hline DES & 31 & Melhorar o desempenho ao longo da tourada à corda. & $100,0^{\star *}$ & 0,0 & 0,0 \\
\hline \multirow{5}{*}{ REG } & 34 & Manter padrão de comportamento equilibrado ao longo das touradas à corda em que participa. & $100,0^{* *}$ & 0,0 & 0,0 \\
\hline & 35 & Melhorar o desempenho de tourada para tourada. & $88,9^{*}$ & 5,6 & 5,6 \\
\hline & 36 & Obter elevado número de touradas à corda com desempenho semelhante nas várias participações. & $100,0^{* *}$ & 0,0 & 0,0 \\
\hline & 37 & Ser conhecido na sociedade civil pelo bom desempenho nas várias touradas à corda. & $94,4^{*}$ & 5,6 & 0,0 \\
\hline & 38 & Participar em várias touradas à corda para o contraste das suas qualidades. & $88,9^{*}$ & 11,1 & 0,0 \\
\hline
\end{tabular}

comportamento do toiro de lide foram de resposta fechada e dividiram-se em 7 grupos descritos na tabela I.

Dos 38 itens relacionados com o comportamento do toiro de lide, 25 pediam a atribuição de um significado positivo ou negativo a outras tantas caraterísticas (tabela II). Outros 7 itens pediam a classificação da importância dos aspetos abordados em cada grupo do inquérito numa escala de 0 a 5 , em que 0 correspondia a nenhuma importância e 5 a extrema importância (figuras 1 e 2). Os 4 ítens restantes eram perguntas de

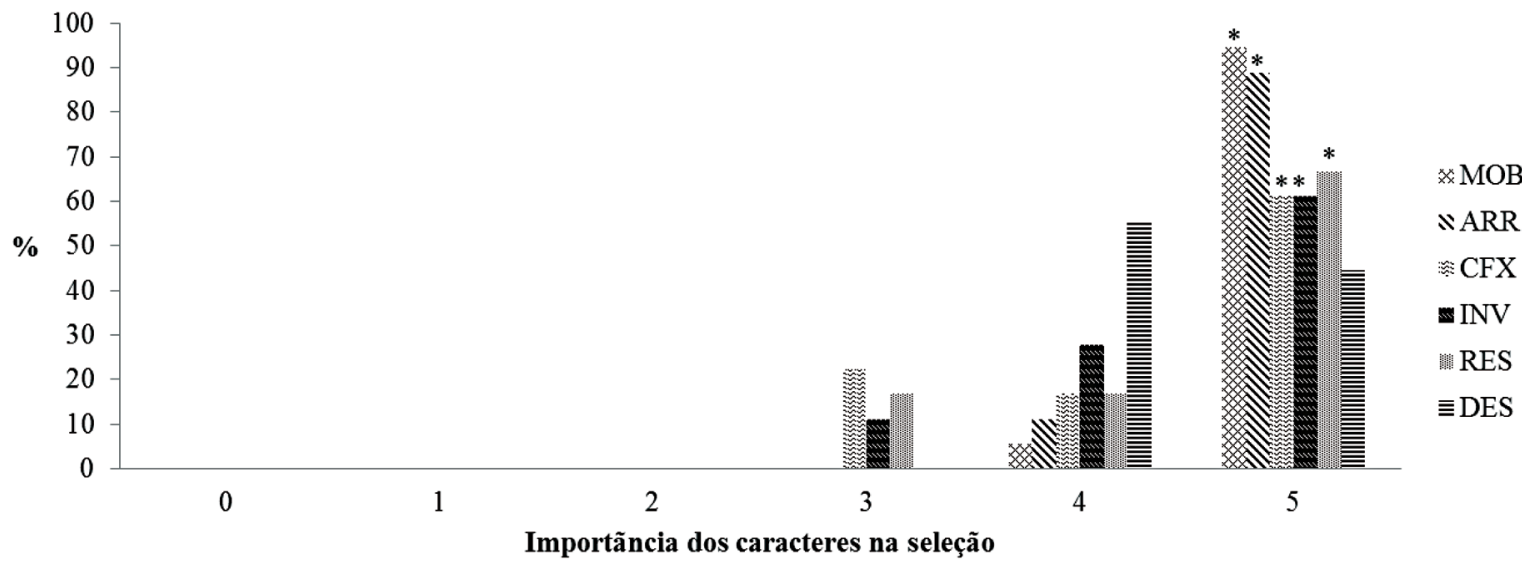

MOB: mobilidade;ARR: capacidade de arremeter; CFX: capacidade de se Fixar; INV: investida; RES: resistência; DES: desempenho ( ${ }^{*}$ pd<0,05).

Figura 2. Importância na seleção de cada característica (MOB5, ARR10, CFX16, INV26, RES30 e DES33); 0= sem importância, $5=$ essencial (Importance levels of the behavioral traits in the selection (MOB5, ARR10, CFX16, INV26, RES30 and DES33), $0=$ unimportant, $5=$ essential). resposta múltipla (figuras 3, 4 e 5). Cada grupo teve entre três e sete questões, identificadas com o acrónimo referente ao capítulo e um número de ordem (e.g. MOB1 ... MOB6).

Os inquéritos realizaram-se num período compreendido entre janeiro e março de 2013 e foram aplicados à totalidade dos ganadeiros $(\mathrm{n}=18)$ inscritos na Associação Regional de Criadores de Toiros da Tourada à Corda, sendo estes os únicos criadores existentes que praticam seleção especifica para a tourada à corda. 


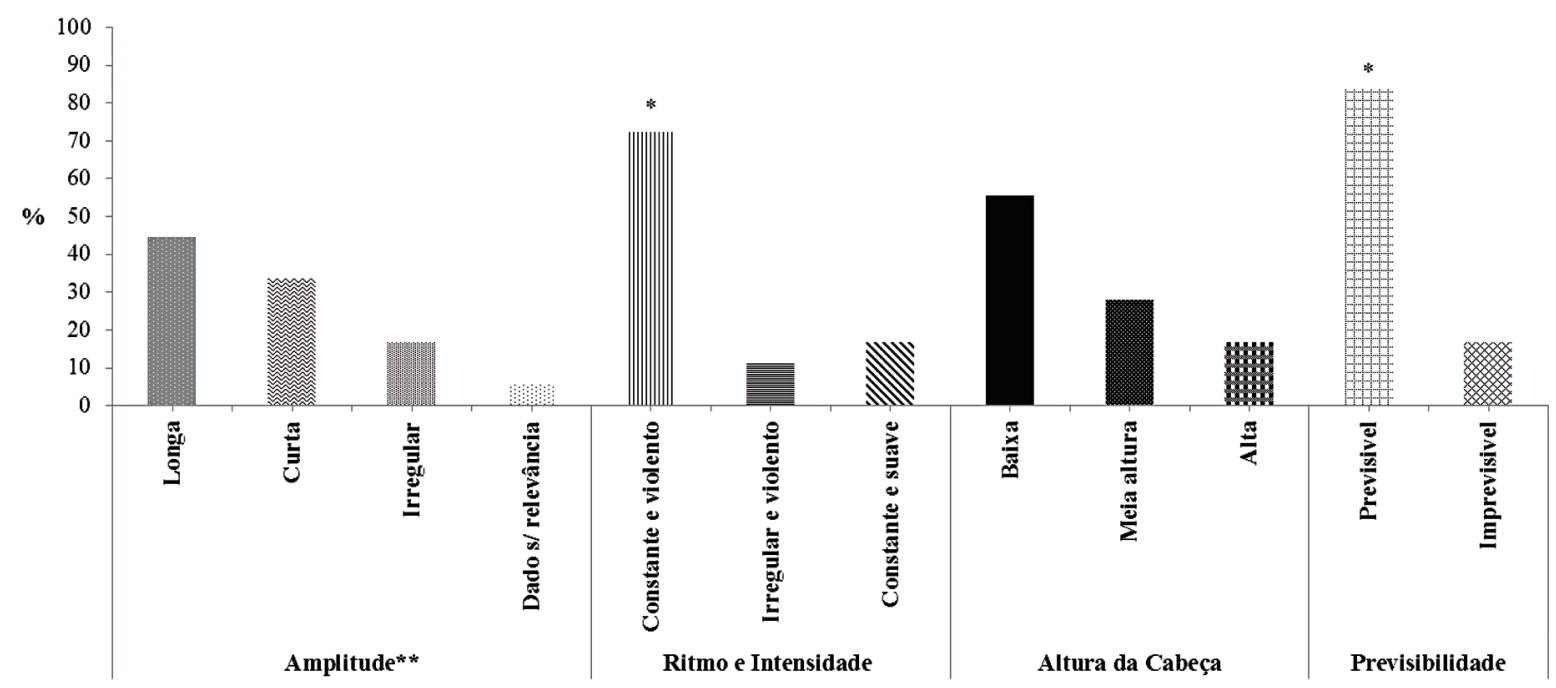

* $p \leq 0,05 ;{ }^{* *}$ Distância entre o início e final da investida.

Figura 3. Formas de investida do toiro na tourada à corda ( INV17: amplitude; INV18: ritmo e intensidade; INV23: altura da cabeça; INV24: previsibilidade) (Attack mode in the Azorean street bullfighting (INV17: amplitude; INV18: rhythm and intensity; INV23: head height; INV24: predictability)).

Os inquiridos estavam dispersos pelas ilhas Terceira $(n=12)$, Graciosa $(n=1)$, São Jorge $(n=4)$ e Pico $(n=1)$.

As respostas ao inquérito foram analisadas em SPSS 17 , com um teste não paramétrico de qui-quadrado por simulação Monte Carlo (MMC-10 000 bootstraps), para um nível de significância de $\mathrm{p} \leq 0,05$.

As respostas que obtiveram diferenças significativas ou mostraram-se constantes em cada grupo, assim como os grupos que obtiveram um grau de importância com pontuações $\geq 3$, permitiu gerar um conjunto de informações para o desenvolvimento de definições dos carateres que tem interesse para a seleção do toiro de lide para a tourada à corda.

\section{RESULTADOS E DISCUSSÃO}

\section{ANÁLISE DEMOGRÁFICA DA POPULAÇ̃̃o}

Do total dos ganadeiros inquiridos (18), o correspondente a $88,89 \%$ (16) eram do sexo masculino e $11,1 \%$ (2) eram do sexo feminino. Os inquiridos, divididos pelas ilhas Terceira (em número de 14, ou representando $77,78 \%$ ), Graciosa (1, ou 5,56 \%), São Jorge (2, ou $11,11 \%$ ) e Pico (1, ou $5,56 \%)$, apresentavam idades compreendidas entre 19 e 71 anos $(47,11 \pm 13,28)$. O $66,67 \%$ dessa população (12 indivíduos) estava vinculada à profissão de empresário agrícola, sendo apenas um dos inquiridos criador de bovinos bravos a tempo inteiro. Os restantes $6(33,33 \%)$ possuíam outra atividade profissional em paralelo à de ganadeiro. Relativamente à escolaridade, observou-se que $1(5,56 \%)$ não detinha qualquer grau de escolaridade, $8(44,44 \%)$ tinham o $1^{\circ}$ ciclo, $4(22,22 \%)$ o $2^{\circ}$ ciclo, $1(5,56 \%)$ o $3^{\circ}$ ciclo, e os restantes $4(22,22 \%)$ possuíam grau secundário (12 $2^{\circ}$ ano). Nenhum dos ganadeiros possuía qualquer curso de nível universitário ou equivalente. Em média, os ganadeiros inquiridos dedicavam-se à atividade de criadores de toiros de lide há 15,28 anos $( \pm 11,65)$, tendo o mais antigo 40 anos de experiência e o mais recente apenas 1 ano.

\section{ANÁLISE DOS INQUÉRITOS}

De um modo geral, os ganadeiros inquiridos mostraram opiniões comuns sobre os caratéres comportamentais mais adequadas para as touradas à corda, permitindo-nos construir definições para essas caraterísticas.

Em relação à intensidade da mobilidade (MOB1), as respostas não foram estatisticamente diferentes e todos os ganadeiros lhe atribuíram pontuação elevada ( $\geq 3$; figura 1), indicando que a mobilidade nunca deverá ser reduzida. O facto de alguns ganadeiros não lhe terem atribuído a pontuação máxima pode dever-se à noção empírica de uma associação entre mobilidade excessiva e falta de atenção dos animais ao que ocorre em seu redor. Esta falta de atenção está muitas vezes relacionada com uma tendência dos toiros para a fuga desenfreada, aspeto depreciado pelos criadores nos seus depoimentos. Por outro lado, a constância das respostas relativas à agilidade e à velocidade $(\mathrm{MOB} 2$ e MOB3), assim como à coordenação de movimentos

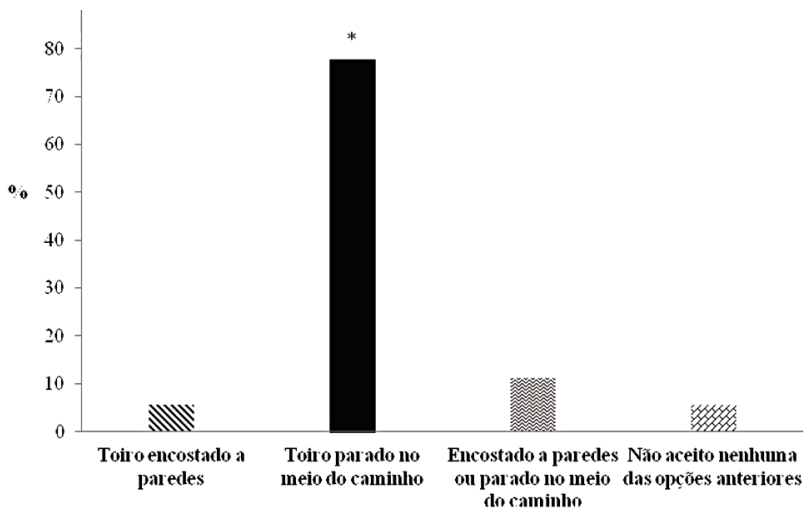

Figura 4. Comportamento aceite pelos ganadeiros quando os toiros apresentam esgotamento físico, evidenciado pela resposta ao item RES29 do inquérito. $\left({ }^{*} \mathrm{pd} \leq 0,05\right)$ (Behavior accepted by breeders when the bulls have physical exhaustion, evidenced by the response to the item $\left.\operatorname{RES} 29\left({ }^{*} \mathrm{p} \leq 0.05\right)\right)$. 


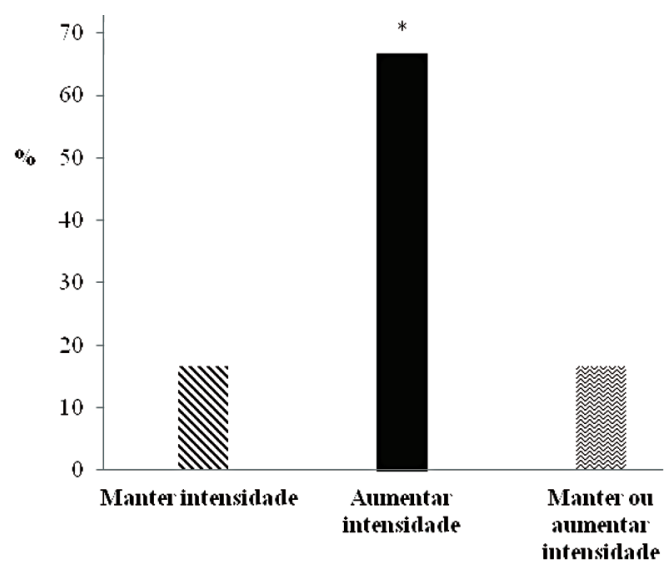

Figura 5. Comportamento da preferência dos ganadeiros em relação à evolução da intensidade das investidas dos toiros nas touradas à corda, evidenciado pela resposta ao item DES32 do inquérito $\left({ }^{*} \mathrm{pd} \leq 0,05\right)$ (Behavior accepted by the breeders, regarding the evolution of the attack endurance in the Azorean street bullfighting evidenced by the item DES32 ( $\left.\left.{ }^{*} \mathrm{p} \leq 0.05\right)\right)$.

(MOB4), leva-nos a inferir que estes são bons indicadores da mobilidade. Tendo em conta que, para medir caraterísticas comportamentais, é necessário definir carateres (Garcia e Almenara 2004; Van der Staay 2006; Lloyd et al., 2007), podemos definir mobilidade, de acordo com os resultados obtidos, como: A capacidade de o animal, durante a tourada à corda, se deslocar de modo intenso, constante e coordenado, sem perder a capacidade de se fixar. Evidenciando a importância da mobilidade do toiro na tourada à corda, $88,89 \%(\mathrm{p} \leq 0,05)$ dos ganadeiros considera que este é um caráter fundamental na seleção (figura 2).

Sobre a capacidade de arremeter, todos os ganadeiros estão de acordo em que os animais têm de responder rapidamente aos estímulos de investida (ARR6; $100,00 \%$ ) (tabela II). Em ARR9, um temperamento altamente agressivo e combativo no toiro é classificado pelos criadores como altamente positivo $(100,00 \%$; tabela II). Sinais como a tentativa de fuga ou a busca de sítios de defesa, como as gaiolas, são vistos como aspetos negativos, como ficou evidenciado pela constância das respostas a ARR 7 e 8 (100,00 \%; tabela II). Perante os resultados observados, podemos definir o caráter capacidade de arremeter como reagir sem hesitação, investindo, a todas as provocações, durante toda a tourada à corda. Esta característica ainda foi apontada pelos ganadeiros como uma das mais importantes na seleção do toiro de lide para a tourada à corda (figura $2=94,44 \%-p \leq 0,05$ ). Tanto as definições apresentadas neste estudo para o caráter mobilidade como capacidade de arremeter são semelhantes às apresentadas no estudo de caracteres da seleção do toiro de lide para as corridas de toiros designadas por movilidad e a cometividad, apresentadas por Gaudioso et al. (1985) e García e Almenara (2004).

Outro dos aspetos estudados foi o da capacidade que os animais têm de fixar o que ocorre no meio que os rodeia. As diferenças significativas encontradas em CFX11, com uma frequência de 94,44 \%, (tabela II), demonstraram que os ganadeiros entendem que os animais devem estar atentos, com a visão e audição, ao que ocorre à sua volta. Por sua vez, aspetos de dis- tração são desprestigiados pelos criadores, tal como evidenciaram os resultados de 100,00 \% em CFX13 e 94,44 \% em CFX15 ( $\leq 00,05)$. Quanto à fixação dos toiros nas capas e guarda-sóis dos capinhas, as divergências de resposta foram maiores que as observadas na questão anterior (CFX12), mesmo que a maioria dos criadores considerasse que o ideal seria que os animais focassem a atenção sobre os vultos que os homens transportam nas mãos. Embora não tenham sido encontradas diferenças significativas, alguns resultados sugerem que existe uma preferência de 72,22 \% pelos toiros que mantêm alguma atenção nos capinhas enquanto investem contra os trastes usados para capear (CFX14). As divergências encontradas relativamente às capacidades de os animais se fixarem em determinados estímulos podem refletir-se negativamente no resultado global da seleção do toiro de lide para a tourada à corda nos Açores. Assim, e atendendo à importância atribuída a este caráter (figura 2), propomos para ele uma definição baseada nos itens que reuniram o maior apoio dos criadores. Propomos, ainda, que esta caraterística passe a ser denominada capacidade de fixar. Ao contrário das caraterísticas anteriormente citadas, o conceito de capacidade de fixar ganha um sentido ligeiramente diferente daquele que é usualmente atribuído ao caráter fijeza na seleção dos animais para as corridas de toiros, onde é privilegiada a atenção sobre os trastes utilizados pelos toureiros (García e Almenara, 2004). Sendo assim, e tendo em conta os resultados obtidos, a capacidade de fixar deverá ser tida em consideração de acordo com a seguinte definição: Capacidade que o animal tem de estar atento ao que ocorre em seu redor durante a tourada à corda.

Em relação às caraterísticas da investida, tais como altura a que o toiro deve meter a cabeça, distância percorrida entre o final do passe e a reposição corporal para o passe seguinte (amplitude da investida) ou derrotar na investida, os criadores voltaram a mostrar algumas divergências. Neste campo, apenas o curvar da cabeça durante a investida foi visto como um aspeto positivo pela maioria dos ganadeiros (INV22=94,44 \%; $\mathrm{p}<0,05)$ (tabela II). Por outro lado, 66,67 \% $(\mathrm{p}<0,05)$ dos ganadeiros manifestaram a opinião de que o toiro na tourada à corda não deve apresentar qualidades de animal toureável (INV25). Desta forma, a seleção do toiro para a tourada à corda não deve ser direcionada para a obtenção de animais que permitam o toureio dentro das suas bases técnicas (parar, templar e mandar). À semelhança da capacidade de fixar, este e outros aspetos diferenciam a seleção dos animais de raça Brava de Lide de acordo com o tipo de festejos, como já era apontado pelo ganadeiro Dinis Fernandes em meados do século XX (Silva, 2011).

Relativamente a outros aspetos inerentes à investida, nomeadamente o temperamento que o toiro nela deve demonstrar, à pergunta INV18, 72,22 \% dos criadores responderam que os animais devem investir em ritmo constante e violento, revelando temperamento agressivo e poder físico (figura 3). A totalidade dos ganadeiros destacou como sinais positivos a repetição da investida e a demonstração de agressividade e codícia, como se vê pelas frequências de 100,00 \% obtidas em INV19 e INV20 (tabela II). Independentemente das 
divergências observadas, os ganadeiros consideram a investida do animal como fundamental para a tourada à corda (figura 2). Este caráter deverá ser identificado com o termo codícia, definido como qualidade do animal que investe de modo agressivo, com força e ânsia de atingir os vultos, curvando a cabeça, independentemente da sua altura, procurando constantemente o que o provoca.

Sendo o toiro o grande responsável pelo dinamismo inerente à tourada à corda, a sua resistência é para os ganadeiros um factor primordial, tendo todos respondido, em RES27 e RES28 (tabela II), que o toiro deve apresentar vigor e resistência ao longo de toda a tourada à corda e, se possível, nunca apresentar esgotamento físico. De acordo com o Decreto Legislativo Regional n. ${ }^{\circ}$ 37/2008/A, o regulamento da tourada à corda exige que um toiro permaneça no arraial um tempo mínimo de 15 minutos e um máximo de $30 \mathrm{mi}$ nutos, o que, segundo Antunes (2002), pode ser carga física em demasia para a natureza do toiro de lide enquanto ruminante. Assim sendo, a fadiga excessiva do toiro de lide, nas touradas à corda, pode surgir com elevada frequência. Relativamente a este caráter, e em caso de esgotamento físico, 77,78 \% dos ganadeiros dão preferência aos animais que ficam parados no centro da estrada, nunca evidenciando sinais de renunciar à luta (RES29; figura 4). No conjunto dos três carateres mais importantes, nomeadamente mobilidade, capacidade de arremeter e resistência, observou-se que este último é o de maior importância, podendo ser definido como a capacidade de suportar a lide, na tourada à corda, sem demonstrar sinais de cansaço ou fadiga.

$\mathrm{Na}$ resposta ao item DES31, todos os ganadeiros consideraram que a melhoria do desempenho do toiro ao longo de uma tourada à corda é um sinal positivo. Além disso, os criadores apontam ainda como sinal positivo a capacidade para investir cada vez com maior pujança ao longo da tourada (DES32; figura 5), o que pode destacar um animal na avaliação do seu desempenho geral. Quanto à importância na seleção, este foi o único caráter classificado maioritariamente com o grau 4 da escala de Likert (figura 2). Por sua vez, a ausência de diferença significativa entre as frequências das classificações 4 e 5 pode estar associada a critérios de seleção distintos entre ganadeiros, como constatou Gaudioso et al. (2001). Esta capacidade de o animal melhorar o seu desempenho ao longo de uma tourada à corda poderá ser denominada crescer. Apesar de este caráter ter uma grande importância na seleção dos animais para a tourada à corda, a sua menor importância relativa pode estar associada a uma maior preocupação de que poderá ser objecto em rebanhos selecionados especificamente para as corridas de toiros (García e Almenara, 2004). Contudo, entendemos que deve ser considerado na seleção do toiro de lide para a tourada à corda, com a seguinte definição: Capacidade de o toiro melhorar o seu de-sempenho durante uma tourada à corda.

Tendo em conta que nas touradas à corda os toiros são corridos várias vezes, gera-se um problema de classificações repetidas para um só animal. No entanto, poderiam ser determinadas as notas que ganham maior consistência nas distintas avaliações que são executadas a cada toiro em cada tourada à corda onde o animal participa, determinando-se assim a classificação final para cada caraterística. No seguimento desta ideia junta-se a caraterística regularidade, que segundo manuscritos de antigos ganadeiros apresentados por Silva (2011), é um dos pontos de diferença entre a seleção do toiro de lide para a lide em praça e tourada à corda. Através dos resultados obtidos, verificou-se que a regularidade é um caráter muito levado em consideração pelos ganadeiros. A totalidade dos inquiridos defende que os toiros devem manter desempenho equilibrado entre as várias touradas à corda em que participam (REG34 e REG36; tabela II).

A regularidade também é considerada importante, por maioria significativa dos inquiridos, para que os animais sejam conhecidos na sociedade (tabela II; $94,44 \%$ das respostas a REG37) e para o contraste das qualidades dos animais (tabela II; $88,89 \%$ das respostas a REG38). No entanto, em REG35, 88,89 \% dos ganadeiros enunciam que o melhorar das suas capacidades com o aumento de touradas também é benéfico. Contudo, a melhoria drástica do desempenho de uma participação para a outra poderá refletir uma grande influência ambiental, visto que estes animais podem acumular experiência (Peláez del Hierro e Vea Baro, 1997; Purroy, 2003). Assim, a melhoria do desempenho de um toiro de tourada para tourada por influência ambiental pode conduzir a erros graves na escolha do macho reprodutor, onde se espera que o fenótipo esteja fortemente associado ao potencial genético. Sendo assim, entendemos que a regularidade deverá ser avaliada pela consistência das notas das restantes 6 caraterísticas comportamentais entre as várias touradas à corda em que os toiros participam.

À semelhança dos métodos de avaliação de parâmetros comportamentais para a seleção do toiro de lide visando a sua utilização em corridas de praça, como o método de Gil-Cabrera (2013) para a avaliação da agressividade, o método francês INRA-COMPAN avaliado por Garcia-Schneider (2008), o de Calvo (2010) e o EBL-10 desenvolvido por Almenara-Barrios e García (2011), um esquema de avaliação dos caracteres definidos neste estudo \% mobilidade, capacidade de arremeter, capacidade de fixar, codícia, resistência, crescer e regularidade $\%$, em escala de intensidade de Likert (figura 6), também pode constituir uma mais-valia para investigadores e ganadeiros na seleção orientada para as touradas à corda dos Açores.

\section{CONCLUSÃO}

Ainda que o presente estudo tenha um número de amostras reduzido, podemos considerar que albergou toda a população de ganadeiros responsáveis pela seleção do toiro de lide para a tourada à corda. Assim sendo, este estudo permitiu definir 7 caraterísticas com interesse na seleção do toiro de lide para a tourada à corda: mobilidade, capacidade de arremeter, capacidade de fixar, codícia, resistência, crescer e regularidade. Esta investigação ganha especial relevância, pois pode afirmar-se como pioneira na documentação das caraterísticas que os ganadeiros procuram incrementar nos toiros de lide utilizados para as touradas à corda. Além disso, este estudo pode ser o primeiro passo para o surgimento de outras investigações que possam desenvol- 


\begin{tabular}{|c|c|}
\hline \multicolumn{2}{|c|}{ Ficha de Avaliação de Caracteres Funcionais para a Tourada à Corda } \\
\hline Ganadaria: & ID Animal: \\
\hline Corda: & Arraial: \\
\hline Tempo: & Avaliador: \\
\hline
\end{tabular}

1. Capacidade de arremeter - Animal que reage sem hesitar, investindo a todas as provocações que recebe, durante toda a tourada à corda.

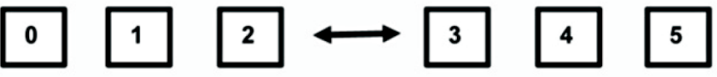

2. Mobilidade - Capacidade do animal deslocar-se de modo intenso, constante e coordenado durante a tourada à corda, sem perder capacidade de se fixar.

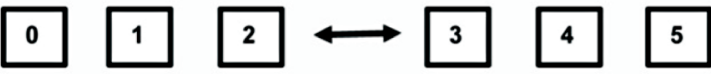

3. Codícia - Animal que investe de modo agressivo, com força e ânsia de atingir os vultos, curvando a cabeça, independentemente da sua altura, procurando o que o provoca constantemente.

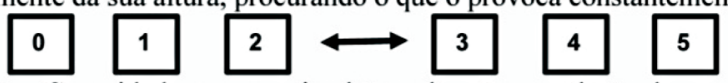

4. Capacidade de fixar - Capacidade que o animal tem de estar pendente do que ocorre em seu redor durante a tourada à corda.

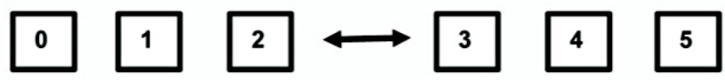

5. Resistência - Capacidade de suporte da lide da tourada à corda sem demonstrar sinais de cansaço ou fadiga.

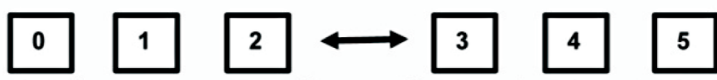

6. Crescer - Capacidade do toiro melhorar o seu desempenho durante o decorrer de uma tourada à corda.

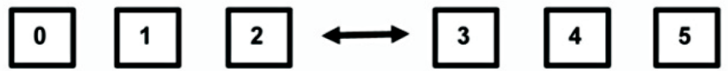

7. Amplitude investida - Distância percorrida durante a investida (longa, média, curta)

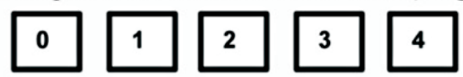

8. Humilhar - Altura a que o animal mete a cabeça (alta, média ou baixa)

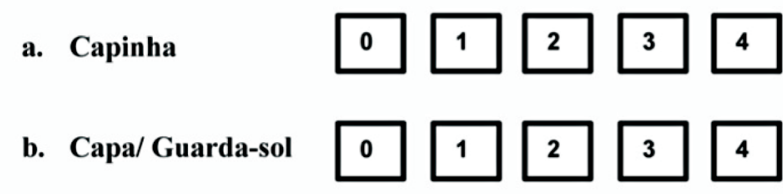

*Característica regularidade determinada pela consistência das pontuações obtidas entre várias touradas à corda onde o animal participa.

Figura 6. Escala para avaliação de caracteres dos toiros nas touradas à corda, onde também são incluídos os parâmetros que medem a amplitude da investida (7) e altura a que os animais colocam a cabeça durante as investidas (Humilhar - 8) com o intuito de caraterizar esses aspetos (Assessment scale for the bulls in Azorean street buIlfighting, which are also included parameters that measure the attack amplitude (7) and head height during the attack (Humilhar- 8) in order to characterize these traits).

ver uma escala válida para o registo de dados que pode contribuir em grande medida para o melhoramento de ganadarias ou associações de criadores.

\section{AGRADECIMENTOS}

Este projeto é suportado por uma bolsa de doutoramento (M3.1.2/F/050/2011) do Fundo Regional da Ciência (FRC). Os nossos agradecimentos são também endereçados ao Centro de Investigação de Tecnologias Agrárias dos Açores (CITA-A).

\section{BIBLIOGRAFIA}

Almenara-Barrios, J. and García, G.R. 2011. Assessment scale for behaviour in bullfighting cattle (EBL 10). Reliability and validity studies. Arch Zootec, 60: 215-224.

Antunes, N.G. 2002. Contribuição para o estudo dos factores ambientais que podem interferir no desempenho físico do toiro bravo durante a lide. Universidade Técnica de Lisboa. UTL. Lisboa.

Bruges, A. 1997. O toiro na ilha Terceira. Breve nota sobre a sua aparição e evolução. Festa na llha, 1: 6-7.

Calvo, L.A. 2010. Determinación evolutiva hasta el siglo XXI de los caracteres morfo-etológicos del Bos taurus braquiceros, subespecie 
lidia. Tesis Doctoral. Facultad de Veterinaria. Universidad Complutense de Madrid.

Costa, G. 1999. A ganadaria brava na ilha Terceira. Festa na Ilha, 3 : 18-22.

Ferreira, A. 2007. Toiros - Casta da Terra. Festa na llha, 11: 45.

García, R.G. y Almenara, J.B. 2004. Escala de evaluación de la bravura para bovinos de lidia (EBL-10). Estudios Taurinos, 18: 251-278.

García, R.; Almenara, J.; Valdés, J.; Navarro, J.I.; Salvador, L. y Fernández, R. 2003. Evaluación estandarizada de la bravura. http:// www.toroslidia.com/2003/05/20/evaluacion-estandariza da-de-labravura-2/ (06/04/2014).

Garcia-Schneider, J.M.N. 2008. Développement et validation d'une nouvelle méthode quantitative et objective d'évaluation du comportement et des dépenses énergétiques du taureau Brave au cours de la corrida: Applications à l'étude de la faiblesse des taureaux lors de la corrida. Thèse d'exercice. Université Paul-Sabatier de Toulouse. Ecole Nationale Vétérinaire de Toulouse. 167 pp.

Gaudioso, V.; Riol, J.A. y Alonso, M.E. 2001. Comportamiento del ganado bravo en el ruedo. In: Purroy y Buxadé (Coord.). II Jornadas sobre ganado de lidia (Ponencias). Ed. Mundi-Prensa. Madrid.

Gaudioso, V.R.; Sánchez, J.M. y Riol, J.A. 1993. Metodología de valoración de la aptitud productiva de lidia. Memoria del I Simposium Nacional del Toro de Lidia. Zafra. Badajoz. pp. 139-149.

Gaudioso, V.R. and Sánchez, J.M. 1987. Influence de la surface par animal sur le comportement agonistique des taureaux. Biol Behav, 12: $239-244$

Gaudioso, V.R.; Pérez-Tabernero, A. y Sánchez, J.M. 1985. Evaluación de la bravura, nobleza y mansedumbre del toro de lidia. Buiatría Española, 1: 218-232.
Gil-Cabrera, F. 2013. Variables neuroendocrinas y su relación con el comportamiento durante la lidia en el toro bravo (Bos taurus, L.). Tesis Doctoral. Universidad Complutense de Madrid. Madrid. 213 pp.

González, C.E.; Durán, C.C.V.y Domínguez, C.J.F. 1994. Heredabilidad y repetibilidad de la nota de tienta y nota de lidia en una ganadería de reses bravas. Arch Zootec, 43: 225-237.

Lloyd, A.S.; Martin, J.E.; Bornett-Gauci, H.L.I. and Wilkinson, R.G. 2007. Evaluation of a novel method of horse personality assessment: Rateragreement and links to behaviour. Anim Behav Sci, 105: 205-222.

Merelim, P. 1986. Tauromaquia terceirense. Ed. Delegação do Turismo de Angra do Heroísmo. pp. 39-40.

Purroy, A. 2003. Comportamiento del toro de lidia en el campo, en el ruedo. Universidad Pública de Navarra. 267 pp.

Peláez del Hierro, F. y Veá i Baró, J.J. 1997. Etología. Bases biológicas de la conducta animal y humana. Colección Psicología. Ediciones Pirámide S.A. Madrid. 400 pp.

Sánchez, J.M.; Riol, J.A.; Castro, M.J. y Gaudioso, V.R. 1990. Comportamiento del toro de lidia frente al caballo y muleta: aspectos aplicativos en la selección de la raza. Arch Zootec, 39: 165-174.

Secretaria Regional da Agricultura e Florestas. 2010. Portaria n ${ }^{\circ} 45 / 2010$ de 6 de Maio de 2010.

Silva, B.; Gonzalo, A. and Cañón, J. 2006. Genetic parameters of aggressiveness, ferocity and mobility in the fighting bull breed. Anim Res, 55: 65-70.

Silva, I.C. 201 1. Ganadaria da ilha Terceira. Casa Agrícola José Albino Fernandes. Portugal.

Van der Staay, J.F. 2006. Animal models of behavioral dysfunctions: Basic concepts and classifications, and an evaluation strategy. Brain Res Rev, 52:131-159. 